\title{
Derivation of the final OSIRIS-REx OVIRS in-flight radiometric calibration
}

\author{
Amy A. Simon ${ }^{\circ},{ }^{a} *$ Dennis C. Reuter, ${ }^{a}$ and Dante S. Lauretta ${ }^{b}$ \\ ${ }^{a}$ NASA Goddard Space Flight Center, Solar System Exploration Division, Greenbelt, \\ Maryland, United States \\ ${ }^{b}$ University of Arizona, Lunar and Planetary Laboratory, Tucson, Arizona, United States
}

\begin{abstract}
The OSIRIS-REx Visible and InfraRed Spectrometer (OVIRS) operates over the $\sim 0.4-$ to $4.3-\mu \mathrm{m}$ wavelength range. Radiometric calibration over this broad range requires the use of multiple calibration sources. Initial OVIRS in-flight calibration coefficients were previously computed using ground calibration from visible integrating sphere and IR blackbody sources, cross-calibrated with Earth-observing satellites for visible wavelengths, and adjusted using the spectrum of the OSIRIS-REx mission's target body, asteroid (101955) Bennu. As part of the final in-flight calibration, we determined that the best calibration method removes out-ofband IR signal leakage prior to radiometric calibration and updated the calibration coefficients accordingly. These final calibration coefficients work well for data where the field of view is filled, and we document the possible artifacts in underfilled spots. (C) The Authors. Published by SPIE under a Creative Commons Attribution 4.0 Unported License. Distribution or reproduction of this work in whole or in part requires full attribution of the original publication, including its DOI. [DOI: 10 .1117/1.JATIS.7.2.020501]
\end{abstract}

Keywords: optics; spectrometer; radiometry; calibration; OSIRIS-Rex; pipeline.

Paper 21008L received Jan. 21, 2021; accepted for publication May 3, 2021; published online May 17, 2021.

\section{Introduction}

The Origins, Spectral Interpretation, Resource Identification, and Security-Regolith Explorer (OSIRIS-REx) Visible and InfraRed Spectrometer (OVIRS) is a point spectrometer constructed for the OSIRIS-REx mission to asteroid (101955) Bennu. OVIRS uses two off-axis parabolic mirrors to produce a collimated beam with a 4-mrad field of view (FOV). ${ }^{1}$ The 0.39 - to $4.28-\mu \mathrm{m}$ spectrum is generated using five linear variable (wedged) filters that cover 0.39 to $0.67,0.65$ to $1.09,1.08$ to $1.80,1.76$ to 2.94 , and 2.85 to $4.28 \mu \mathrm{m}$. Deriving radiometric calibration coefficients over this broad wavelength range requires multiple calibration sources. Initial ground characterization was performed using a visible/near-IR integrating sphere and a blackbody flood source, both of which are NIST-traceable. ${ }^{2}$ Observations of these sources were acquired at multiple instrument temperatures and under vacuum to replicate in-flight conditions. The blackbody source provided calibrated, uniform radiance for source temperatures from 270 to $360 \mathrm{~K}$ with an emissivity of $0.992 .^{2}$ The integrating sphere produces uniform radiance from fiber-fed high-temperature lamps, whose output is converted to absolute radiance using sphere-internal calibrated detectors. Ideally, this results in absolute calibration of the OVIRS system response across all wavelengths. However, due to fiber absorption and transmission issues in the integrating sphere, accurate calibration data were not provided in several wavelength ranges in the visible/NIR wavelength region and from $\sim 1.5$ to $2.5 \mu \mathrm{m}$.

After the OSIRIS-REX spacecraft launched in 2016, an Earth-gravity assist was used as an opportunity to better calibrate the short wavelengths using near-simultaneous data obtained by Earth-observing imagers: the Earth Observing System Aqua/Moderate Resolution Imaging Spectroradiometer, Suomi-NPP/Visible Infrared Imaging Radiometer Suite, and DSCOVR/ Earth Polychromatic Imaging Camera. ${ }^{2}$ This cross-comparison corrected the calibration at wavelengths shorter than $2 \mu \mathrm{m}$ by $20 \%$ to $50 \%$ and removed absorption bands caused by the integrating sphere's fibers. However, the Earth's radiance drops off and could not be used to

*Address all correspondence to Amy A. Simon, amy.simon@nasa.gov 
calibrate the wavelengths from 2 to $2.5 \mu \mathrm{m}$. In addition, the Earth data were obtained primarily over low-reflectance, deep ocean regions, resulting in low signal for both OVIRS and Earthobserving satellites, leaving larger-than-desired uncertainty in the derived values. On approach to Bennu in 2018, spectra were obtained in which the FOV was underfilled; the calibration coefficients were adjusted by $30 \%$ to $50 \%$ in the $2.5-\mu \mathrm{m}$ region to ensure a smooth continuous spectrum. The shortest wavelengths were also adjusted by $\sim 10 \%$ to match the ground-based spectral slope previously observed for Bennu. ${ }^{3}$

However, once disk-resolved spectra of Bennu were obtained, several issues were noted in this initial in-flight calibration, primarily appearing as spectral discontinuities where the wavelengths in each filter overlap. This occurred because underfilled FOV data were used to adjust the calibration, and because the instrument operates warmer in flight than anticipated, affecting long-wavelength sensitivity. Both conditions affect the radiometry and also can contribute to improper correction of out-of-band IR signal leakage. The calibration at this point exceeded the science requirement for measuring a 5\% absorption feature at $4 \%$ surface reflectance. However, Bennu's surface is darker than $4 \%$ with few deep spectral signatures. ${ }^{4}$ Thus, the calibration needed to be adjusted to accurately address the actually observed absorption features of $\leq 2 \%$ at high phase angle where the reflectance was $<1.5 \%$. In this paper, we discuss the method used to determine the final in-flight radiometric calibration coefficients and how the calibration pipeline order was changed to better address the feedback between radiometric calibration and the out-of-band signal removal. We also illustrate remaining issues in calibrating and using spectra that have an underfilled FOV or high solar phase angle. Lastly, we list steps that future users of the data could implement to improve the usability of off-nominal data sets.

\section{Mission Phases and Datasets}

OSIRIS-REx is a NASA New Frontiers-class asteroid sample return mission. To adequately survey Bennu and select a sample site, the mission was separated into phased observing campaigns. ${ }^{5,6}$ On approach in November 2018, full-disk OVIRS spectra were obtained for light curve and global spectrum analyses. The first resolved OVIRS data, from Preliminary Survey in December 2018, were largely acquired at high phase angles with partial surface coverage. The main surface mapping phase, Detailed Survey, occurred in March to June 2019. In this mission phase, 10 observing sets included OVIRS data: three Baseball Diamond (BBD) stations designed for mosaics and other instrument coverage, and seven equatorial (EQ) stations, each at a different local solar time, where global OVIRS coverage was obtained. After potential sample collection sites were identified, the Reconnaissance phase began in October 2019 with a series of lower altitude flyovers of the four candidate locations. Progressively lower-altitude flyovers of the final primary site and backup location occurred in early 2020. Each mission phase resulted in tens of thousands of spectra (Table 1) with additional data sets acquired between some mission phases. Over the life of the primary mission, OVIRS acquired over 500,000 Bennu spectra.

Table 1 OVIRS data sets by mission phase.

\begin{tabular}{llc}
\hline \hline Mission phase & \multicolumn{1}{c}{ Dates } & Bennu spectra \\
\hline Approach & November 2 to December 2, 2018 & 103,995 \\
Preliminary survey & December 4 to December 16, 2018 & 86,572 \\
Detailed survey (BBD) & March 7 to March 21, 2019 & 52,067 \\
Detailed survey (EQ) & April 25 to June 6, 2019 & 152,836 \\
Detailed survey BBD2 Refly & September 26, 2019 & 8,227 \\
Reconnaissance A & October 5 to October 26, 2019 & 16,395 \\
Reconnaissance B & January 21 to February 12, 2020 \\
Reconnaissance C & March 3 to May 21, 2020 & 23,009 \\
\hline \hline
\end{tabular}




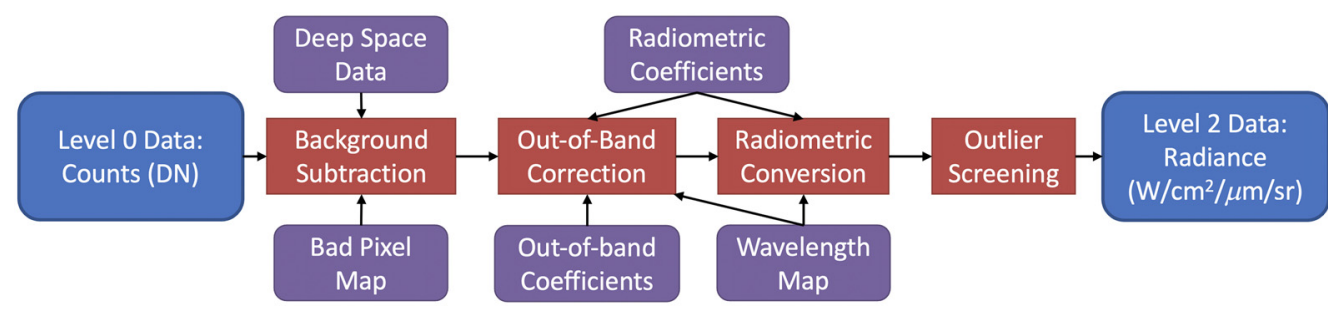

Fig. 1 Calibration pipeline flow diagram. Each step of the calibration process requires ancillary files; some are acquired with the science data (deep space data), while others are produced from ground and in-flight calibration sources (calibration coefficients and pixel maps).

\section{Calibration Pipeline}

An automated data ingest system and subsequent calibration pipeline were developed to allow rapid data validation and to enable the science team to quickly produce the data products and analysis necessary to proceed to the next mission phase. For example, Detailed Survey data were used to produce global maps, identify potential sample sites, and aid in selection of the four locations that would be further surveyed in the Reconnaissance phase. The OVIRS calibration pipeline converts the raw data (level 0 ) from detector counts to physical units (level 2 radiance, $\mathrm{W} / \mathrm{cm}^{2} / \mathrm{sr} / \mu \mathrm{m}$ ), Fig. 1 . The processing steps are: (1) subtract the average background (dark current and thermal emission) level and apply the bad pixel map (which removes the contribution of poorly operating pixels from the summed pixel signal), (2) calculate and remove out-of-band signal contribution, (3) convert to radiance units, and (4) screen and remove $3 \sigma$ outlying pixels. The details of each of these steps are documented with the calibrated data delivered to the Planetary Data System. ${ }^{7}$ We will now explain the out-of-band removal process.

\subsection{Calculating Out-of-Band Contributions}

Out-of-band signal occurs when photons whose wavelengths are well outside of the nominal filter bandwidth at a given position are transmitted through the filter at that position. The filters were designed, and exceed, a requirement to provide average out-of-band blocking to $0.01 \%$ and peak out-of-band blocking to better than $0.5 \%$. Models of the filter transmission characteristics and the spectral radiance distribution indicate that, by far, the primary out-of-band contribution arises from the sum of long-wavelength contributions to the signal in the short-wavelength filter segments. Although a small effect (a few percent), ${ }^{2}$ it is most noticeable when the surface is hot (large IR signal) and the surface reflectance is low (small visible wavelength signal). For OVIRS, this effect is noted in all filters except the longest-wavelength filter $(2.85$ to $4.28 \mu \mathrm{m})$, Fig. 2 . To correct for this leakage, the background-subtracted science counts are first converted to photon radiance (photon $\left./ \mathrm{s} / \mathrm{cm}^{2} / \mathrm{sr} / \mu \mathrm{m}\right)$. The photon radiance of pixel $(x, y), P_{x, y}$, is given as

$$
P_{x, y}=\frac{C_{x, y} R_{x, y}}{t * E_{x, y}},
$$

where $C_{x, y}$ is the background-subtracted counts for the pixel, $R_{x, y}$ is the radiometric conversion coefficient for that pixel, $t$ is the integration time, and $E_{x, y}$ is the photon energy at the wavelength of that pixel:

$$
E_{x, y}=\frac{h c}{\lambda_{x, y}}
$$

where $h$ is the Planck constant, $c$ is the speed of light, and $\lambda_{x, y}$ is the wavelength of the pixel.

Next, the photon radiance is integrated under the curve from 2.8 to $3.9 \mu \mathrm{m}$ using the trapezoidal rule. The summed long-wavelength photon radiance is then converted to an out-of-band signal (in counts) at each shorter-wavelength pixel using correction factors computed from the calibrated blackbody source data acquired in ground testing. The out-of-band counts are then 


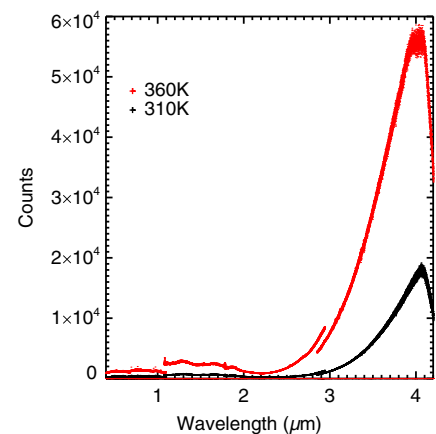

(a)

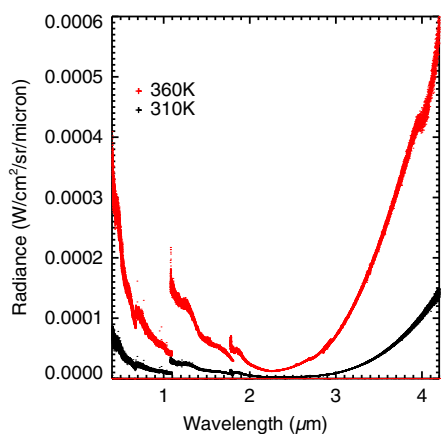

(b)

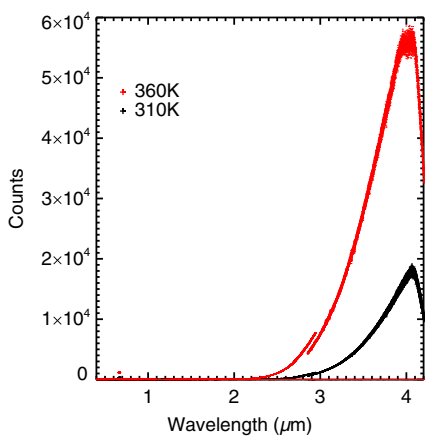

(c)

Fig. 2 Ground test blackbody data and out-of-band subtraction. (a) Blackbody curves at $310 \mathrm{~K}$ (black) and $360 \mathrm{~K}$ (red), at the raw instrument count level. The 360-K curve shows saturation above $3.9 \mu \mathrm{m}$, a flattening of the signal at the maximum 16-bit value ( 58,000 counts with detector bias and background levels). (b) Curves from (a), converted to radiance units, showing the spectral shape of the out-of-band signal. (c) Data from (a) after out-of-band signal is subtracted and before radiometric calibration.

removed from the first four filters' background-subtracted counts, Fig. 2(c), before proceeding to final radiometric calibration, Fig. 1.

\subsection{Initial In-flight Radiometry Derivation}

As a point spectrometer, OVIRS has limited astronomical calibration targets; there are no stars or other sources visible to the instrument that can be used as a reference standard. Onboard blackbody and filament sources are used for performance trending but not absolute radiometric calibration. ${ }^{2}$ Similarly, a solar calibration port allows for absolute flux trending, but it does not have an identical optical path to the primary science beam and cannot be used for direct radiometric calibration. ${ }^{1}$ Although the Earth flyby improved the radiometry, the lack of signal above $\sim 2 \mu \mathrm{m}$ led to nonphysical features in Bennu's radiance spectrum when first observed and calibrated.

Bennu's spectrum is a combination of reflected solar signal and thermal radiance from the surface. To refine the Earth-flyby-derived radiometric coefficients on approach, a model spectrum was generated using the solar flux at Bennu's heliocentric range, assuming a uniform surface reflectance of $4 \%$. The thermal component was derived from the approach data using a single temperature blackbody and an emissivity of 0.96 . This model was used to smooth the radiometric coefficients between 2 and $3 \mu \mathrm{m}$. As the initial calibration pipeline performed the out-of-band subtraction after the radiometric conversion at all wavelengths, all corrections were made to the radiometric coefficients. ${ }^{2}$

\section{Final In-flight Calibration}

As noted, the initially derived in-flight out-of-band and radiometric coefficients did not result in clean spectra when applied to resolved Bennu data. Iterations to both the calibration steps and coefficients were required to properly calibrate OVIRS flight data because of incorrect assumptions about the early Bennu data and the out-of-band removal. First, the early approach data only filled $\sim 40 \%$ of the OVIRS FOV, resulting in a blackbody fit that incorrectly modeled the thermal flux that causes the out-of-band signal. Second, the calibration pipeline performed the out-ofband correction after the radiometric conversion, but because the initial in-flight radiometric coefficients were adjusted to generate a smooth, overlapping, Bennu approach spectrum, they were also partially correcting for out-of-band contributions in the data. As these incorrect radiometric coefficients were then used to calculate out-of-band corrections from the ground test data, new discontinuities resulted when applied to the filled FOV data. Although the initial in-flight calibration was checked against the Earth data, those spectra were much brighter than Bennu and the thermal flux lower, effectively eliminating the out-of-band contribution. 


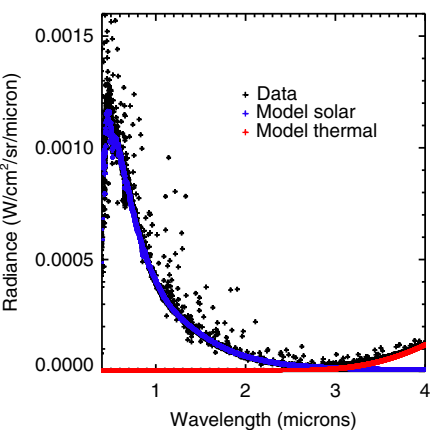

(a)

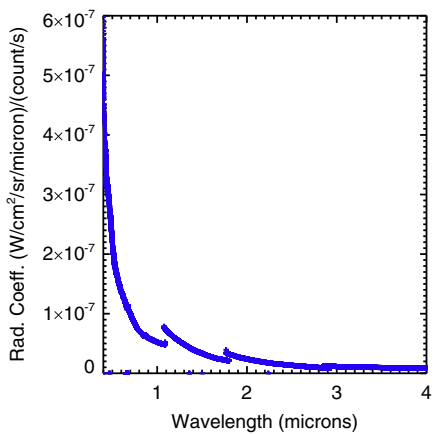

(b)

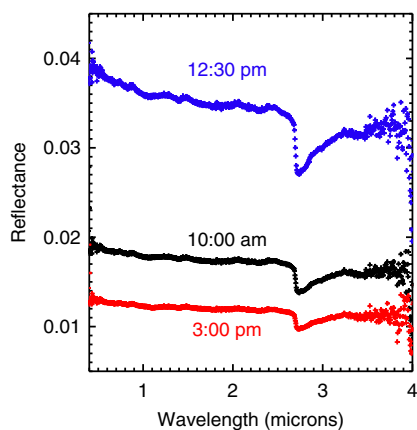

(c)

Fig. 3 Radiometric model and resulting calibration. (a) An example radiometric model that matches 30 deg phase angle data, using a reflected solar component and a 313.5-K thermal component. (b) Final derived radiometric coefficients. (c) Average of several hundred reflectance spectra (thermal tail-removed radiance divided by solar flux) from 10:00 am, 12:30 pm, and 3:00 pm Bennu data.

To correct the observed radiometric issues, several changes were implemented in the pipeline and to the calibration files. First, the pipeline step order was reordered so that out-of-band subtraction is performed at the background-subtracted count level. This removes the feedback between the out-of-band correction and radiometry at short wavelengths and is possible because the longest-wavelength radiometric coefficients were very accurately determined from ground test data acquired at OVIRS's flight operational temperatures. ${ }^{2}$ Next, the model solar plus thermal spectrum was adjusted by approximating the Bennu reflectance as measured by the OSIRISREx Camera Suite, OCAMS, ${ }^{8}$ at similar phase angles, Fig. 3(a).

Globally averaged, resolved, low-phase-angle Bennu spectra were then compared with the model spectrum to adjust the radiometry in all filters except the longest wavelengths, while meeting the requirement for a continuous spectrum. Because the resolved Bennu data show spectral features, the radiometric coefficients were also directly examined to ensure that no artifacts were introduced, as they should smoothly vary within each filter, Fig. 3(b). Lastly, the new coefficients were tested on Earth data and higher-phase-angle Bennu spectra to confirm that they show continuous spectra and expected radiances, Fig. 3(c). This derived radiometric approach allowed reliable detection of spectral features with absorption band depths $<1 \%$, much smaller than the $5 \%$ instrument requirement. ${ }^{1,4,9}$

\section{Remaining Data Issues}

The final calibration approach is optimized for relatively low phase angles $<45$ deg and performs well for the great majority of the data sets, but there are artifacts observed in some spectra, especially those acquired in off-nominal instrument or viewing conditions. The two main residual calibration issues are spectral jumps and residual out-of-band effects and both can be present in the same data. The more frequent artifact, the spectral jump, occurs when the OVIRS footprint crosses sharp illumination boundaries, for example, a large shadowed region or the limb of a body, Fig. 4(a), and is due to the OVIRS design.

The OVIRS spectrum is generated from five separate filter segments placed across the detector with physical separation. Although all pixels see collimated light, the readout rolls down the detector array and all pixels are not read out at the same time. The two shortest-wavelength filters are positioned on opposite ends of the detector array, and all spectra were acquired with the spacecraft nodding (to produce ground tracks for spatial coverage). During the integration time and readout, the filters can potentially be exposed to slightly different regions on the ground track when their pixels are read out. Near a sharp boundary, this can result in one filter receiving more illumination than the other when the pixels under it are read out, and this manifests as an entire segment of the spectrum having a much lower or higher signal than the adjacent filters. This artifact is most prominent at high phase angles where the contribution of reflected solar radiation to the observed signal is very low. 


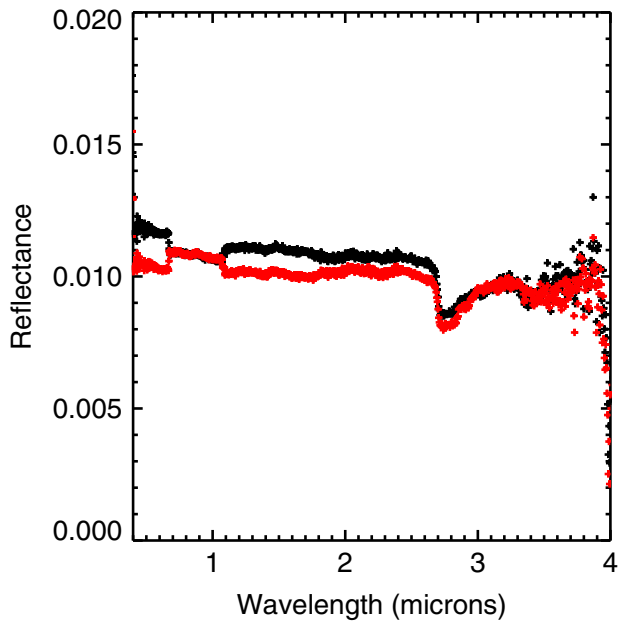

(a)

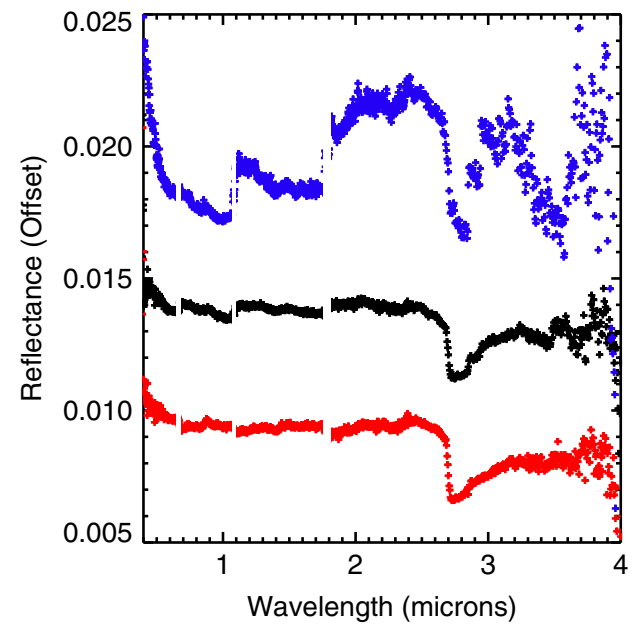

(b)

Fig. 4 Residual calibration artifacts in reflectance spectra. (a) Filter segment jumps from 0.65 to $1.09 \mu \mathrm{m}$ in adjacent spectra, resulting from crossing an illumination boundary. (b) Cases of poor out-of-band subtraction with filter overlap regions removed for clarity. The effect can be a minor under correction (black curve) or overcorrection (red curve, offset by -0.008). In extreme cases, false spectral signatures can also appear (blue curve), particularly near $1.8 \mu \mathrm{m}$.

Poor out-of-band correction can also occur in some circumstances, Fig. 4(b). The out-of-band correction is somewhat dependent on the assumption of a blackbody distribution of IR radiance; the correction coefficients were computed assuming a linear fit to blackbody spectral curves at several temperatures $(310$ and $360 \mathrm{~K})$. As the OVIRS detector is operating near the upper end of its expected temperature range, ground data sets were limited, and a multiparameter fit to the out-of-band signal (to account for scenes that deviate from a blackbody shape) is not possible. In addition, during some observations the instrument was further heated, particularly when the spacecraft was close to Bennu's surface. As the OVIRS detector gets warmer, it loses sensitivity at long wavelengths and integration over the observed thermal signal does not accurately represent the actual integrated flux; it underestimates the out-of-band signal correction. The effect on the spectrum is twofold; the spectrum becomes noncontinuous, with each filter segment showing increased signal toward shorter wavelengths, and in extreme cases spectral artifacts can also appear, mirroring the detailed shape of the out-of-band signal. In rare cases, the outof-band signal may be overcorrected, if a very small hot area dominates the FOV. Incorrect out-of-band subtraction is most often seen in underfilled FOV data, or when the instrument was very warm during Reconnaissance $\mathrm{B}$ and $\mathrm{C}$ observations.

\subsection{Artifact Solutions}

The filter segment jumps can be potentially corrected in a number of ways. First, adjacent alongtrack spectra can be averaged, to compensate for the illumination boundary. This works well when the problem filter segment appears low in one spectrum and high in the adjacent spectrum, but averaging spectra does reduce the spatial resolution. Another solution is to treat each filter segment separately in time and location, but this requires detailed knowledge of the instrument pointing and timing, treating each segment as a separate observation, and computing and storing five times more geometric information for each spectrum; such information is not currently available. A practical approach that has proved useful is to manually bring the wavelength segment into alignment with the rest of the spectrum before analysis.

Improperly subtracted out-of-band signal is more difficult to correct. To first order, the spectra with poor out-of-band subtraction can be made more continuous in the same manner as the segment jumps, but because the out-of-band also imparts spectral features, Fig. 2, it is not straightforward to remove. In principle, further fits to the ground test data might yield improvements, as could further testing on Moon data collected during the Earth flyby, which also did not 
fill the FOV. A more nuanced method would be to use the Approach data, with the more accurate radiometry and Bennu's known surface temperatures, to attempt a multiparameter fit. This would work best if multiple data sets with different phase angles are used to replicate different mixedtemperature scenes. Each of the discussed correction methods are too complicated to implement in an automated pipeline but might be manually applied.

\section{Summary}

The OVIRS instrument has broad spectral range that presented several challenges to absolute radiometric calibration. Using a combination of NIST-traceable ground sources, Earth flyby data, and Bennu data, we have refined the calibration procedure and radiometric calibration coefficients to provide accurate radiances for the majority of the OVIRS data. The radiometric coefficients were adjusted using knowledge of Bennu at low solar phase angles, and the derived calibration works well in most circumstances. Future users of the data may be able to further refine data taken in off-nominal conditions, such as over sharp illumination changes or at high phase angles, to fix spectrum discontinuities at filter wavelength boundaries. Data taken at these very off-nominal conditions are still usable and will not be reprocessed with the operational pipeline.

\section{Acknowledgments}

We are grateful to the entire OSIRIS-REx Team for making the encounter with Bennu possible and especially thank Victoria Hamilton, Ellen Howell, and Beth Clark for feedback on the OVIRS data and calibration quality. We also acknowledge Allen Lunsford for initial pipeline development, Nicolas Gorius for code assistance, and Richard Cosentino for data trending. This material is based on work supported by NASA under Contract NNM10AA11C issued through the New Frontiers Program. Disclosures: The authors declare no relevant financial interests in the manuscript and have no other potential conflicts of interest to disclose.

\section{Data Availability}

All OVIRS spectral data collected during the mission, both uncalibrated (level 0, raw counts) and calibrated (level 2, radiance), as well as the calibration files, are available via the Planetary Data System at: https://sbn.psi.edu/pds/resource/orex/ovirs.html. ${ }^{10}$ Data calibrated with the updated pipeline are denoted by calv2 in the filename.

\section{References}

1. D. C. Reuter et al., "The OSIRIS-REx Visible and InfraRed Spectrometer (OVIRS): spectral maps of the asteroid Bennu," Space Sci. Rev. 214, 54 (2018).

2. A. Simon et al., "In-flight calibration and performance of the OSIRIS-REx Visible and IR Spectrometer (OVIRS)," Remote Sens. 10, 1486 (2018).

3. B. E. Clark et al., "Asteroid (101955) 1999 RQ36: spectroscopy from 0.4 to 2.4 and meteorite analogs," Icarus 216, 462-475 (2011).

4. D. DellaGiustina et al., "Exogenic basalt on asteroid (101955) Bennu," Nat. Astron. 3, 341 (2020).

5. D. S. Lauretta et al., "OSIRIS-REx: sample return from asteroid (101955) Bennu," Space Sci. Rev. 212, 925-984 (2017).

6. D. S. Lauretta et al., OSIRIS-REx at Bennu: Overcoming Challenges to Collect a Sample of the Early Solar System, Elsevier, in press (2021).

7. D. Reuter et al., "Calibration description: OSIRIS-REX visible and infrared spectrometer (OVIRS),” tech. rep., NASA Planetary Data System, https://sbnarchive.psi.edu/pds4/orex/ orex.ovirs/document/ovirs_cal_doc_v3.0.pdf (2020).

8. B. Rizk et al., "OCAMS: The OSIRIS-REx camera suite," Space Sci. Rev. 214, 26 (2018). 
9. A. A. Simon et al., "Widespread carbon-bearing materials on near-Earth asteroid (101955) Bennu," Science 370, eabc3522 (2020).

10. D. Reuter et al., "Origins, spectral interpretation, resource identification, security, regolith explorer (osiris-rex): visible and infrared spectrometer (OVIRS) bundle," tech. rep., NASA Planetary Data System, urn:nasa:pds:orex.ovirs (2019). 